\title{
Lymph node pathology using optical spectroscopy in cancer diagnostics
}

\author{
M. Isabelle ${ }^{\mathrm{a}, *}$, N. Stone ${ }^{\mathrm{a}}$, H. Barr ${ }^{\mathrm{b}}$, M. Vipond ${ }^{\mathrm{b}}$, N. Shepherd ${ }^{\mathrm{c}}$ and K. Rogers $^{\mathrm{d}}$ \\ ${ }^{a}$ Biophotonics Research Group, Cranfield Health, Gloucestershire Hospitals NHS Foundation Trust, \\ Gloucester, UK \\ ${ }^{\mathrm{b}}$ Gastrointestinal Surgical Unit, Gloucestershire Hospitals NHS Foundation Trust, Gloucester, UK \\ ${ }^{\mathrm{c}}$ Department of Pathology, Gloucestershire Hospitals NHS Foundation Trust, Gloucester, UK \\ ${ }^{\mathrm{d}}$ Department of Materials and Medical Sciences, Cranfield University, Shrivenham, UK
}

\begin{abstract}
Raman and infrared spectroscopy are optical spectroscopic techniques that use light scattering (Raman) and light absorption (infrared) to probe the vibrational energy levels of molecules in tissue samples. Using these techniques, one can gain an insight into the biochemical composition of cells and tissues by looking at the spectra produced and comparing them with spectra obtained from standards such as proteins, nucleic acids, lipids and carbohydrates. As a result of optical spectroscopy being able to measure these biochemical changes, diagnosis of cancer could take place faster than current diagnostic methods, assisting and offering pathologists and cytologists a novel technology in cancer screening and diagnosis.

The purpose of this study is to use both spectroscopic techniques, in combination with multivariate statistical analysis tools, to analyze some of the major biochemical and morphological changes taking place during carcinogenesis and metastasis in lymph nodes and to develop a predictive model to correctly differentiate cancerous from benign lymph nodes taken from oesophageal cancer patients.

The results of this study showed that Raman and infrared spectroscopy managed to correctly differentiate between cancerous and benign oesophageal lymph nodes with a training performance greater than $94 \%$ using principal component analysis (PCA)fed linear discriminant analysis (LDA). Cancerous nodes had higher nucleic acid but lower lipid and carbohydrate content compared to benign nodes which is indicative of increased cell proliferation and loss of differentiation.

With better understanding of the molecular mechanisms of carcinogenesis and metastasis together with use of multivariate statistical analysis tools, these spectroscopic studies will provide a platform for future development of real-time (in surgery) non-invasive diagnostic tools in medical research.
\end{abstract}

Keywords: FTIR, Raman spectroscopy, lymph node, metastasis, cancer, oesophagus

\section{Introduction}

Cancer is a disease of the cells in the body where normal cell division has become uncontrolled and unregulated through mutations in the cell's DNA which leads to the production of a growth or lump of tissue made up of these abnormal tumours cells. These tumour growths can become malignant (cancers) gaining the ability to spread to nearby tissues and organs by invasion and metastasis. Cancer cells from this growth may also break off from the primary tumour and invade the bloodstream or lymphatic vessels to be carried to other parts of the body to multiply and form secondary tumours (metastases). The most

\footnotetext{
*Corresponding author: Martin Isabelle, Biophotonics Research Group, Gloucestershire Hospitals NHS Foundation Trust, Great Western Road, Gloucester, GL1 3NN, UK. Tel.: +44 8454 225482; Fax: +44 8454 225485; E-mail: m.isabelle@ medicalresearch-centre.com.
} 
common places for metastases, in addition to lymph nodes, are the adrenals, lungs, liver, brain and bones [1]. The progression of a primary tumour to formation of a secondary tumour or metastasis carries a worse prognosis and can be associated with a poor survival in most cancers [2,3]. The first site of metastasis is usually the lymph nodes near the primary tumour called regional lymph nodes which makes lymph nodes sampling and pathological examination important in assessing whether a primary tumour has spread or has the potential to spread to distant sites to develop secondary tumours [4]. However, current conventional lymph node histopathology staging and grading relies on a morphological analysis by a pathologist and is subject to significant inter- and intra-observer variation [5]. There is a need for a more sensitive and objective tool in lymph node diagnostics.

Raman and infrared spectroscopy are optical techniques that can be used to interrogate biological tissues in order to understand the changes in the molecular constituents that are associated with cancer progression from normal to malignant during carcinogenesis. These spectroscopic techniques have the ability to provide detailed biochemical information since the spectrum obtained from cellular and tissue measurements are intrinsic molecular fingerprint of the sample that reveals information about the DNA, carbohydrate, protein and lipid content [6].

Raman spectroscopy is based on the inelastic scattering of photons by molecular bond vibrations where the shift towards lower frequencies is measured as a small portion of the incident photons are scattered by interaction with the bonds of molecules in the tissue. Infrared spectroscopy is a form of absorption spectroscopy that detects these molecular bond vibrations within a sample via interrogation of the sample using the mid-infrared region of light where the loss of energy or frequency from the incident light is measured as it is absorbed by the molecules in the tissue. Raman and infrared spectroscopy can be used together to obtain more biochemical information from the tissue since certain chemical bonds are more sensitive in Raman than in infrared and vice-versa. For example, hydroxyl stretching, amine stretching and carbonyl groups are usually stronger in FTIR while carbon-carbon double and triple bonds and aromatic symmetrical vibrations are stronger in Raman.

Raman spectroscopic studies have been used to differentiate between different pathologies in a number of cancer types including colon [7,8], oesophageal [9], brain [10], skin [11] and laryngeal [12] cancers as well as lymph nodes [13]. Smith et al. [13] used axillary lymph nodes from breast cancer patients, to demonstrate a correct classification for cancerous nodes with $81 \%$ sensitivity and $97 \%$ specificity using Raman spectroscopy.

Infrared spectroscopic studies have been used to differentiate between different pathologies in skin [14], cervical [15], colorectal [16], leukaemia [17] and prostate cancers [18].

This study has been set up to use both spectroscopic techniques, in combination with multivariate statistical analysis tools, to analyse some of the major biochemical and morphological changes taking place during carcinogenesis and metastasis in lymph nodes and to develop a predictive model to correctly differentiate cancerous from benign lymph nodes taken from oesophageal cancer patients.

\section{Materials and methods}

\subsection{Tissue preparation for spectroscopic analysis and histopathology}

Lymph node samples were selected and removed during oesophagectomies to remove the primary tumours from patients, who consented to donation of lymph nodes for spectroscopic studies. Lymph node samples were cut in half, where one half was placed in formalin and sent for routine histopathology 
(paraffin-embedded sectioning and standard tissue processing) while the other half was snap-frozen in liquid nitrogen and stored in $-80^{\circ} \mathrm{C}$ freezers for spectroscopic analysis.

Frozen unstained sections were prepared of $15 \mu \mathrm{m}$ thickness using a cold cryotome and were mounted on a calcium fluoride $\left(\mathrm{CaF}_{2}\right)$ slide. Prior to spectroscopic measurements, these frozen sections were allowed to defrost and air-dry for at least $45 \mathrm{~min}$.

\subsection{Raman and infrared microspectroscopy}

Raman spectra were collected using a Renishaw ${ }^{\circledR}$ System 1000 Raman microspectrometer consisting of a spectrometer coupled to a diode laser (laser output of $350 \mathrm{~mW}$ and tuned to $830 \mathrm{~nm}$ to reduce autofluorescence from tissue), a Leica ${ }^{\circledR}$ microscope with a condenser, a Prior ${ }^{\circledR}$ electronic stage, a video viewer and a desktop computer with customised Grams ${ }^{\circledR}$ software and WiRE (Windows based Raman Environment) software (version 1.3.15). Twenty to thirty point spectra were measured at 20 seconds, in a single accumulation with an $80 \times$ objective from each tissue section. Image maps $(20 \mathrm{~s}, 1$ accumulation, $100 \mu \mathrm{m}$ steps) were performed on the tissue sections for morphological comparison with haematoxylin and eosin stained sections as well as spectroscopic comparison with FTIR hyper-spectral maps.

Infrared spectra were collected using a Perkin Elmer ${ }^{\circledR}$ Spotlight 300 Fourier transform infrared (FTIR) spectroscopy system in transmission mode. The system has a liquid nitrogen cooled linear MCT array detector, a video camera attached to a microscope to view optical images and a programmable computerized $x-y-z$ stage. An aperture size of $30 \times 30 \mu \mathrm{m}$ was used to collect $20-30$ point spectra (64 samplebackground rationed co-scans) within the wave number range $720-4000 \mathrm{~cm}^{-1}$ with a spectral resolution of $4 \mathrm{~cm}^{-1}$. Image maps (64 sample-background rationed co-scans, aperture size of $100 \times 100 \mu \mathrm{m}$ ) within the wave number range $720-4000 \mathrm{~cm}^{-1}$ with a spectral resolution of $4 \mathrm{~cm}^{-1}$ were performed on the tissue sections for morphological comparison with haematoxylin and eosin stained sections as well as spectroscopic comparison with Raman hyper-spectral maps.

\subsection{Data processing and statistical analysis}

All data analysis was performed using Matlab $7^{\circledR}$ software.

\subsubsection{Point spectra}

Infrared point and map spectra were converted to absorbance using: $(\log (\max (\operatorname{transmittance}))-$ $\log ($ transmittance $))$, where transmittance $=I_{\text {out }} / I_{\text {in }}$.

Raman spectra were energy sensitivity-corrected using the green glass calibration spectrum and interpolated to 1 point per wavenumber.

Both Raman and infrared spectra were smoothed using 2nd order Savitzky-Golay filter to remove short term variations or noise in the spectral dataset, area normalized, for correction of uniform intensity changes by removing sources of systematic variation (e.g. differing amounts and thickness of sample) between spectra.

Cancerous and benign mean spectra were generated from all cancerous point spectra and spectra from all benign point spectra.

\subsubsection{Map spectra}

Raman and infrared map spectra were converted and loaded into Matlab to generate a 3D hyperspectral matrix for each section and then principal component analysis (PCA) was performed to generate PCA pseudo colour maps. Pixel correction, using in-house scripts, was then performed on the Raman PCA score maps to remove remaining cosmic rays and saturated spectra. 
Regions of interest were identified using the H\&E stained section and corresponding features were identified by anatomical landmarks on the unstained tissue sections. These regions were identified and selected on the pseudo colour maps in order to generate a spectral dataset for each tissue section to represent the lymph node's overall tissue pathology spectra.

This combined spectral dataset was then normalized, mean-centred (by calculating the average spectrum of the data set and subtracting that average from each spectrum) and inputted into a PCA-fed linear discriminant analysis (LDA) algorithm in order to assess the training performance for each spectroscopic type in distinguishing the different pathology types (i.e. benign or cancerous).

\section{Results}

In total, 78,624 spectra were collected from the 20 lymph node tissue sections (11 cancerous and 9 non-cancerous nodes confirmed by histopathology).

Analysis of the mean pathology spectra for benign and cancerous (as illustrated in Fig. 1) show there
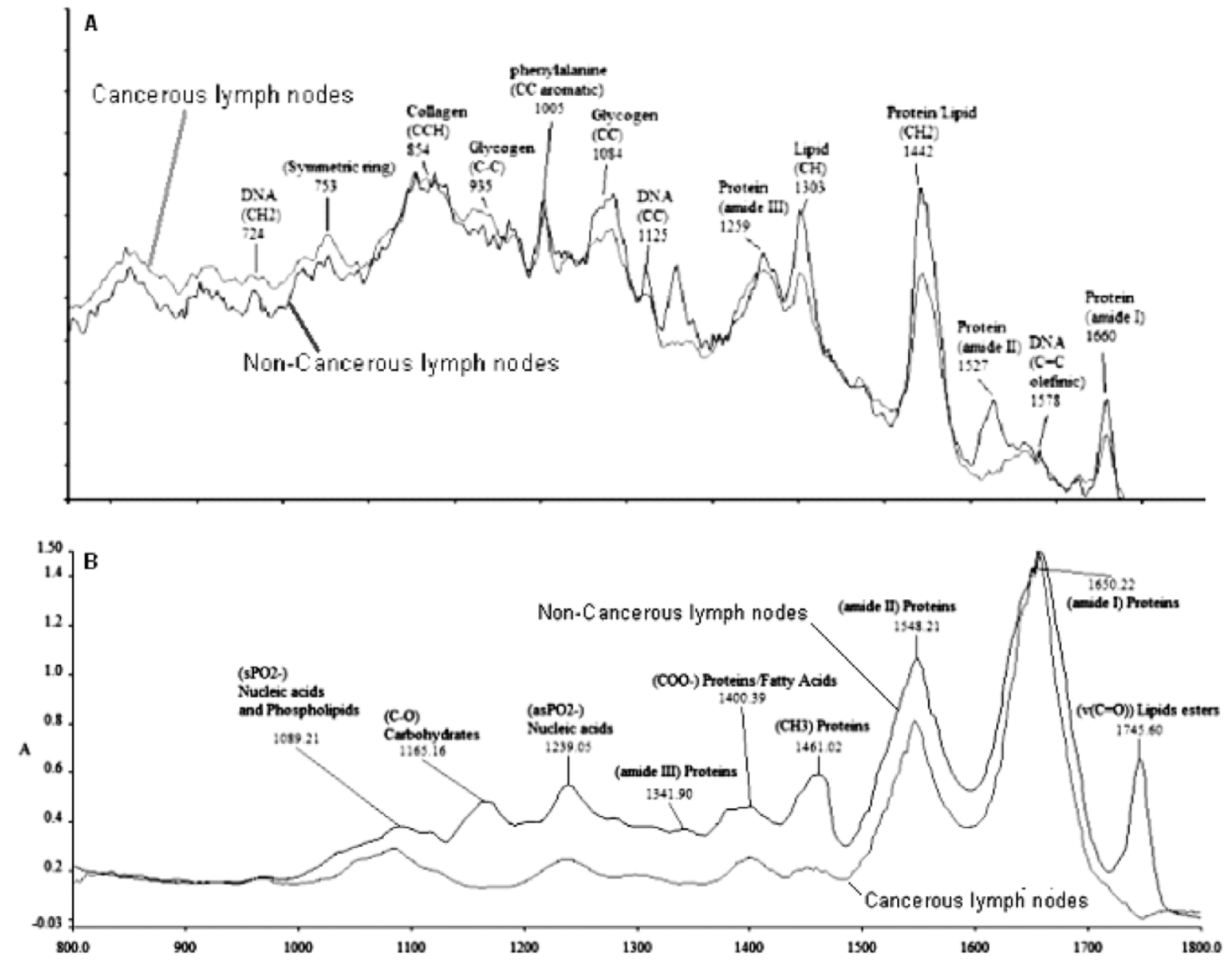

Fig. 1. (A) Raman mean spectra from benign (non-cancerous) and cancerous lymph nodes; (B) infrared mean spectra from benign (non-cancerous) and cancerous lymph nodes. 
are biochemical differences between the spectra of the two pathology types for infrared and Raman spectroscopy.

Benign lymph nodes have higher lipid (Raman: $1303(\mathrm{CH}), 1442\left(\mathrm{CH}_{2}\right)$ [19,20]; infrared: $1745(\mathrm{C}=\mathrm{O}$ lipid ester), $1400\left(\mathrm{COO}^{-}\right)$[21]) and carbohydrate (Raman: 1084 (CC); infrared: 1165 (C-O), 1400 $\left(\mathrm{COO}^{-}\right)$) content compared to cancerous nodes demonstrating that lipids and carbohydrates are being used as a fuel source during carcinogenesis to provide energy for rapid cell proliferation of tumor cells. The increase of nucleic acid peaks (Raman: $724\left(\mathrm{CH}_{2}\right), 1125(\mathrm{CC})$ ) in the Raman spectra indicates that there is an increase in nuclear-to-cytoplasmic ratio and mitotic activity in cancerous tissue as observed by Mahadevan-Jansen and Richards-Kortum in 1996 [23]. The asymmetrical (as $\mathrm{PO}_{2}^{-}$) [1240] and symmetrical $\left(\mathrm{sPO}_{2}^{-}\right.$) [1089] [23] phosphodiester peaks seen in nucleic acid spectra are lower in cancerous tissue however these phosphodiester peaks are also present in the spectra for phospholipids as well. Therefore the higher levels of these peaks could be an indication of higher content of phospholipids, rather than nucleic acids, in non-cancerous nodes.

After generating the absorbance maps for the infrared map spectra and average intensity maps for Raman map spectra, comparison of these with the images from the H\&E section showed that they can provide visual separation of tissue type layers (e.g. cortex, medulla and capsule) seen in the H\&E sections (as illustrated in Fig. 2).

Applying PCA-fed linear discriminant analysis to the spectral dataset resulted in a training performance of $98 \%$ for infrared spectra and $94 \%$ for Raman spectra (as illustrated in Fig. 3). Cross-validation, at this stage, was not attempted due to the small sample size.

\section{Discussion}

This initial study has shown that both infrared and Raman micro-spectroscopy when combined with multivariate statistical analysis tools such as PCA-fed LDA analysis are able to discriminate noncancerous or benign from cancerous lymph node tissue with high performance results and demonstrates that both methods have promising clinical and pathological applications. These training performances are based on a small study with limited number of lymph nodes and once the remaining lymph nodes $(\sim 150)$, that have been collected for this project, are measured and included in the prediction model, better performances and analysis can be performed and elucidation of further biochemical changes between the pathology states can be identified.

After collecting and measuring additional lymph node spectra, a cross-validation-PCA-fed LDA analysis can be performed to provide sensitivities and specificities for pathology prediction.

The analysis of the mean pathology spectra generated from the spectral data demonstrates it is possible to identify biochemical changes during carcinogenesis. By using Raman and infrared spectroscopy combined, more biochemical information can be elicited from the tissue. However, as observed by others [24-26], there are discrepancies in the literature regarding peak assignments and it has been observed that most peaks have contributions from more than one biochemical component [27] resulting in biochemical changes through peak assignment to be often speculative. Therefore multivariate statistical applications, such as ordinary and non-negative least squares biochemical fitting, needs to be applied so that biochemical analysis of the whole spectra at once can be made rather than use individual peak assignments. Our group has already made attempts in biochemical analysis using non-negative least squares fitting for prostate tissue [28], bladder tissue [29], aortic tissue [30] and oesophageal tissue [31]. 

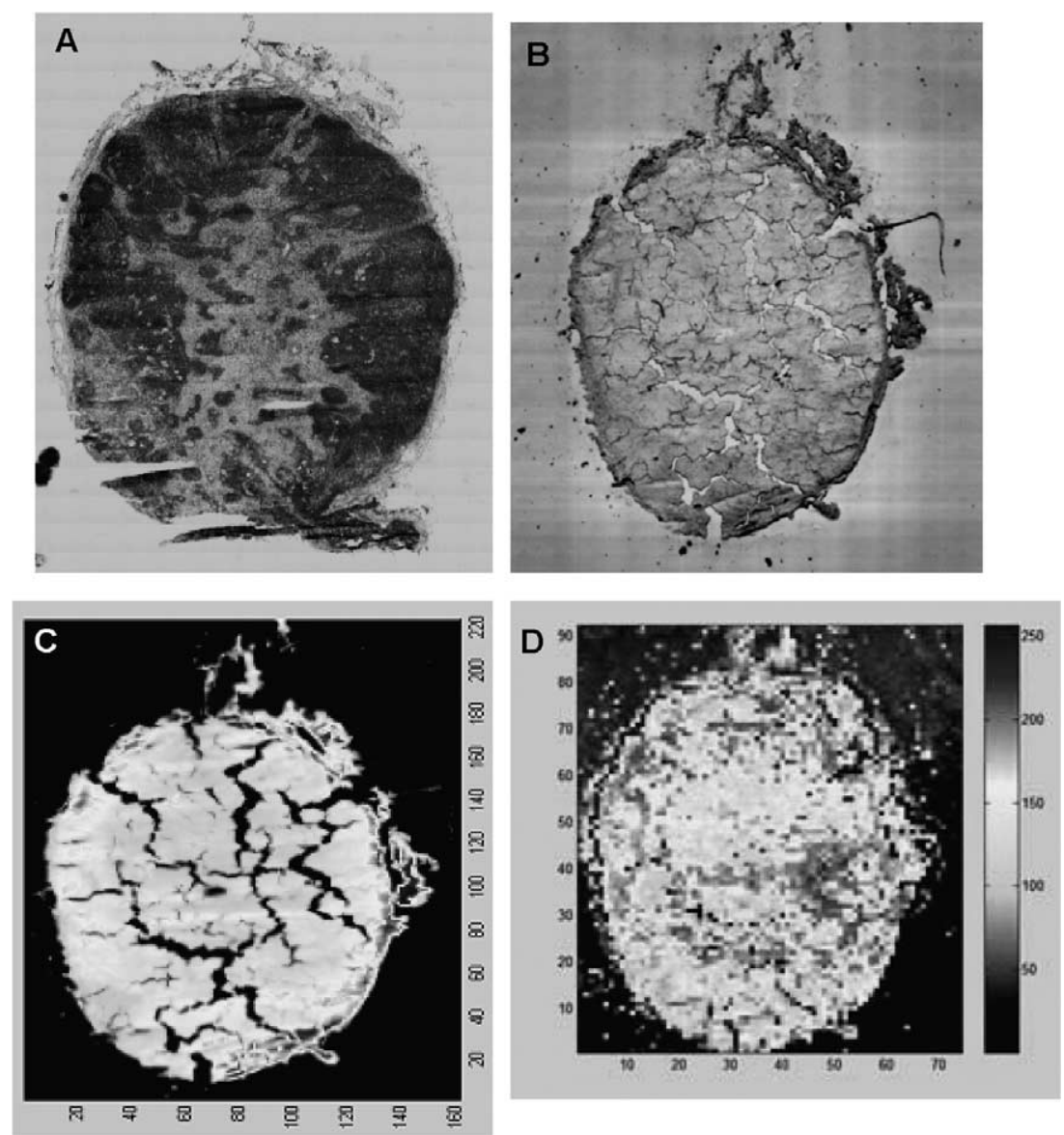

Fig. 2. Benign lymph node. (A) Image of hematoxylin and eosin (H\&E) stained slide; (B) image of the unstained section slide, (C) IR absorbance map ( $800-1800 \mathrm{~cm}^{-1}$ spectral range, $4 \mathrm{~cm}^{-1}$ spectral resolution, $25 \mu \mathrm{m}$ spatial resolution, 64 coscans (400 MB file)); (D) Raman intensity map (450-1850 $\mathrm{cm}^{-1}$ spectral range, $3.75 \mathrm{~cm}^{-1}$ spectral resolution, $55 \mu \mathrm{m}$ spatial resolution, $20 \mathrm{~s}$ per map point (100 MB file)).

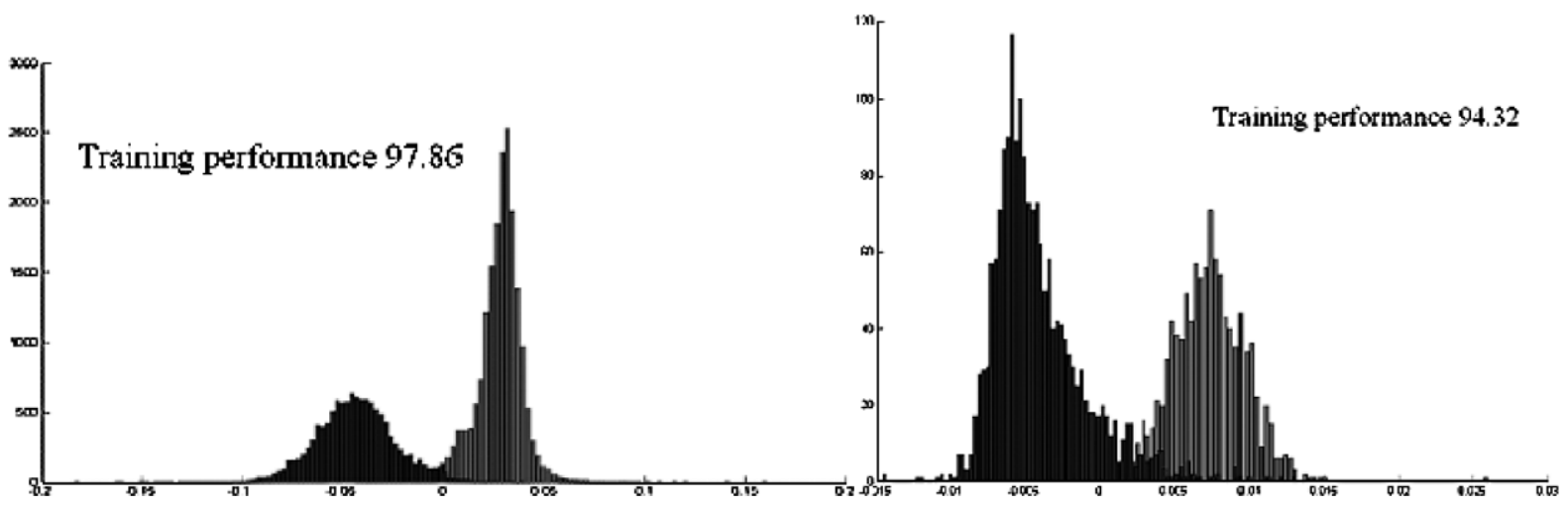

(a)

(b)

Fig. 3. (a) Histogram of linear discriminant scores for benign (non-cancerous) and cancerous lymph nodes for infrared spectroscopy; (b) histogram of linear discriminant scores for benign (non-cancerous) and cancerous lymph nodes for Raman spectroscopy (black: benign; grey: cancerous). 


\section{Acknowledgments}

I would like to thank all contributors to this study including members of Cranfield University and Gloucestershire Hospitals NHS Trust for their financial and logistical support.

\section{References}

[1] National Cancer Institute, Cancer statistics. Metastatic cancer, http://www.cancer.gov/cancertopics/factsheet/SitesTypes/metastatic (accessed on 5th December, 2007).

[2] International (Ludwig) Breast Cancer Study Group, Prognostic importance of occult axillary lymph node micrometastases from breast cancers, Lancet 335 (1990), 1565.

[3] C. Collin, J. Godbold, S. Hajdu and M.F. Brennan, Localised extremity soft tissue sarcoma: an analysis of factors affecting survival, J. Clin. Oncol. 5 (1987), 601-602.

[4] W.E. Sumner, 3rd, M.I. Ross, P.F. Mansfield, J.E. Lee, V.G. Prieto, C.W. Schacherer and J.E. Gershenwald, Implications of lymphatic drainage to unusual sentinel lymph node sites in patients with primary cutaneous melanoma, Cancer 95(2) (2002), 354-360.

[5] B.F. Burns, W.T.E. McCaughey, W. Comrie and A.R. Willan, Observer variation in the pathologic diagnosis of malignant lymphoma in Canada, Cancer 62(2) (2006), 314-318.

[6] J.W. Chan, D.S. Taylor, T. Zwerdling, S.M. Lane, K. Ihara and T. Huser, Micro-Raman spectroscopy detects individual neoplastic and normal hematopoietic cells, Biophys. J. 90(2) (2006), 648-656.

[7] M.S. Feld, R. Manoharan, J. Salenius, J. Orenstein-Carndona, T.J. Romer, J.F. Brennan et al., in: Advances in Fluorescence Sensing Technology II. The International Society for Optical Engineering (SPIE), Vol. 2388, J.R. Lakowicz, ed., Bellingham, 1995, pp. 99-105.

[8] M. Shim, L.M. Song, N.E. Marcon and B.C. Wilson, In vivo near-infrared Raman spectroscopy: Demonstration of feasibility during clinical gastrointestinal endoscopy, J. Photochem. Photobiol. 72 (2000), 146-150.

[9] C. Kendall, N. Stone, N. Shepherd, K. Geboes, B. Warren, R. Bennett and H. Barr, Raman spectroscopy, a potential tool for the objective identification and classification of neoplasia in Barrett's oesophagus, J. Pathol. 200 (2003), 602-609.

[10] B. Schrader, S. Keller, T. Lochte, S. Fendel, D.S. Moore, A. Simon and I. Sawatzki, J. Mol. Struct. 348 (1995), $293-296$.

[11] P. Caspers, G.W. Lucassen, R. Wolthuis, H.A. Bruining and G.J. Puppels, Biospectroscopy 4 (1998), S31-S39.

[12] N. Stone, P. Stravroulaki, C. Kendall, M. Birchall and H. Barr, Raman spectroscopy for early detection of laryngeal malignancy: Preliminary results, Laryngoscope 110 (2000), 1756-1763.

[13] J. Smith, C. Kendall, A. Sammon, J. Christie-Brown and N. Stone, Raman spectral mapping in the assessment of axillary lymph nodes in breast cancer, Technol. Cancer. Res. Treat. 2(4) (2003), 327-332.

[14] L.M. McIntosh et al., Infrared spectra of basal cell carcinomas are distinct from non-tumor-bearing skin components, J. Invest. Dermatol. 112 (1999), 951-956.

[15] M.J. Walsh, M.J. German, M. Singh, H.M. Pollock, A. Hammiche, M. Kyrgiou, H.F. Stringfellow, E. Paraskevaidis, P.L. Martin-Hirsch and F.L. Martin, IR microspectroscopy: potential applications in cervical cancer screening, Cancer Lett. 246 (2007), 1-11.

[16] V.R. Kondepati, M. Keese, R. Mueller, B.C. Manegold and J. Backhaus, Application of near-infrared spectroscopy for the diagnosis of colorectal cancer in resected human tissue specimens, Vibr. Spectrosc. 44(2) (2007), 236-242.

[17] S. Boydston-White, T. Gopen, S. Houser, J. Bargonetti and M. Diem, Infrared spectroscopy of human tissue. V. Infrared spectroscopic studies of myeloid leukemia (ML-1) cells at different phases of the cell cycle, Biospectroscopy 5(4) (1999), $219-227$.

[18] E. Gazi, M. Baker, J. Dwyer, N.P. Lockyer, P. Gardner, J.H. Shanks, R.S. Reeve, C.A. Hart, N.W. Clarke and M.D. Brown, A correlation of FTIR spectra derived from prostate cancer biopsies with Gleason grade and tumour stage, Eur. Urol. 50 (2006), 750-761.

[19] J. Kneipp, T. Bakker Schut, M. Kliffen, M. Menke-Pluijmers and G. Puppels, Characterization of breast duct epithelia: A Raman spectroscopic study, Vibr. Spectrosc. 32 (2003), 67-74.

[20] N. Uzunbajakava, J. Greve and G. Otto, Raman microscopy of cells: Chemical imaging of apoptosis, Proceedings of SPIE - The International Society for Optical Engineering 4963 (2003), 223-230.

[21] S. Neviliappan, L. Fang Kan, T. Tiang Lee Walter, S. Arulkumaran and P.T.T. Wong, Infrared spectral features of exfoliated cervical cells, cervical adenocarcinoma tissue, and an adenocarcinoma cell line (SiSo), Gynecol. Oncol. 85(1) (2002), 170-174.

[22] H.P. Buschman, G. Deinum, J.T. Motz, M. Fitzmaurice, J.R. Kramer, A. Van Der Laarse, A.V. Bruschke and M.S. Feld, Raman microspectroscopy of human coronary atherosclerosis: Biochemical assessment of cellular and extracellular morphologic structures in situ, Cardiovasc. Pathol. 10(2) (2001), 69-82. 
[23] A. Mahadevan-Jansen and R.R. Richards-Kortum, Raman spectroscopy for the detection of cancers and precancers, J. Biomed. Opt. 1(1) (1996), 31-70.

[24] J.R. Mourant, K.W. Short, S. Carpenter, N. Kunapareddy, L. Coburn, T.M. Powers and J.P. Freyer, Biochemical differences in tumorigenic and nontumorigenic cells measured by Raman and infrared spectroscopy, J. Biomed. Opt. 10(3) (2005), 031106.

[25] D.M. Haaland, H.D.T. Jones and E.V. Thomas, Multivariate classification of the infrared spectra of cell and tissue samples, Appl. Spectrosc. 51 (1997), 340-345.

[26] N. Stone, C. Kendall, J. Smith, P. Crow and H. Barr, Raman spectroscopy for identification of epithelial cancers, Faraday Discuss. 126 (2004), 141-157.

[27] J.R. Mourant, Y.R. Yamada, S. Carpenter, L.R. Dominique and J.P. Freyer, FTIR spectroscopy demonstrates biochemical differences in mammalian cell cultures at different growth stages, Biophys. J. 85 (2003), 1938-1947.

[28] J.J. Aning, M. Isabelle, J. Uff, A.W.S. Ritchie, H.W. Gilbert and N. Stone, FTIR biochemical imaging of the prostate: an in-vitro proof of concept study, in: Conference Proceedings 2007, SPIE, 2007, p. 6628-15.

[29] N. Stone, M.C. Hart Prieto, P. Crow, J. Uff and A.W. Ritchie, The use of Raman spectroscopy to provide an estimation of the gross biochemistry associated with urological pathologies, Anal. Bioanal. Chem. 387(5) (2007), 1657-1668.

[30] M.G. Sowa, M.S.D. Smith, C. Kendall, E.R. Bock, C.-T.K.O. Alex, L.-P. Choo-Smith and N. Stone, Semi-parametric estimation in the compositional modeling of multicomponent systems from Raman spectroscopic data, Appl. Spectrosc. 60(8) (2006), 877-883.

[31] G. Shetty, C. Kendall, N. Shepherd, N. Stone and H. Barr, Raman spectroscopy: Elucidation of biochemical changes in carcinogenesis of oesophagus, Br. J. Cancer 94(10) (2006), 1460-1464. 


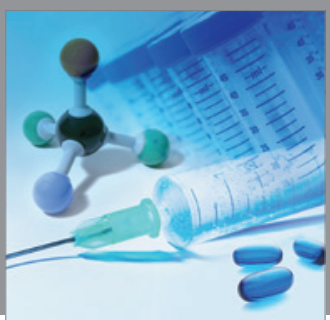

International Journal of

Medicinal Chemistry

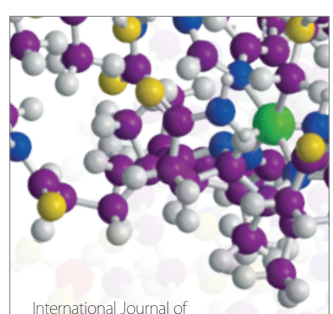

Carbohydrate Chemistry

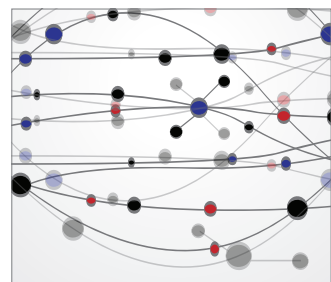

The Scientific World Journal
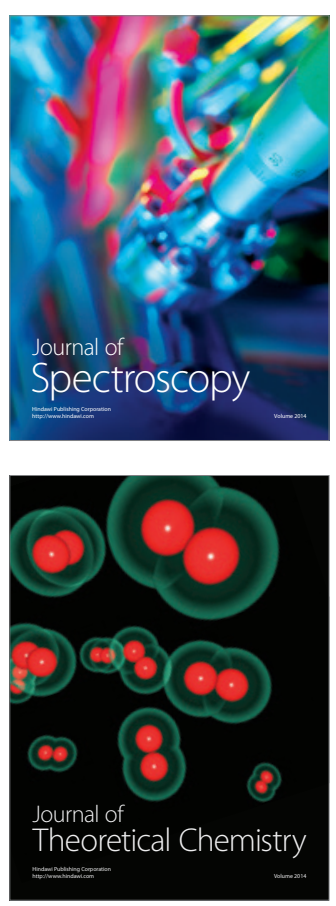
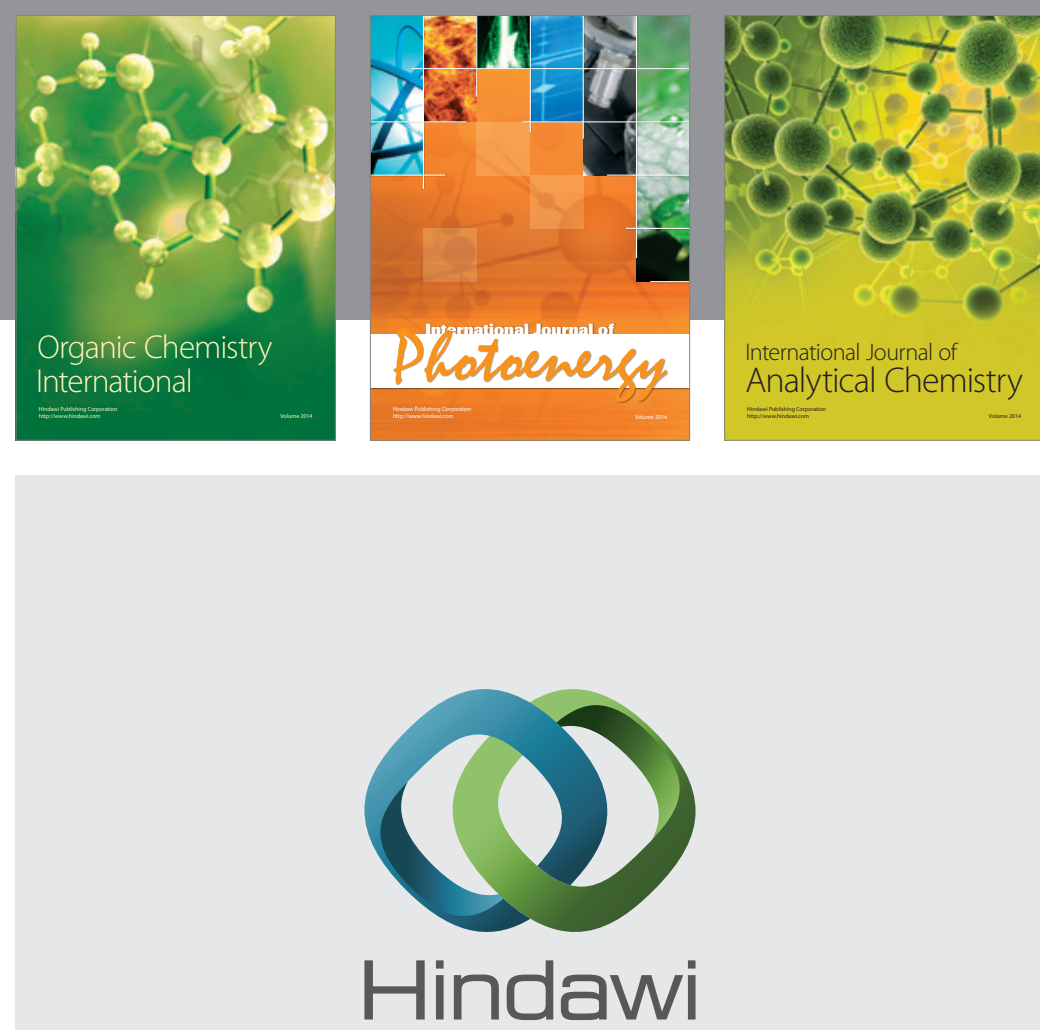

Submit your manuscripts at

http://www.hindawi.com
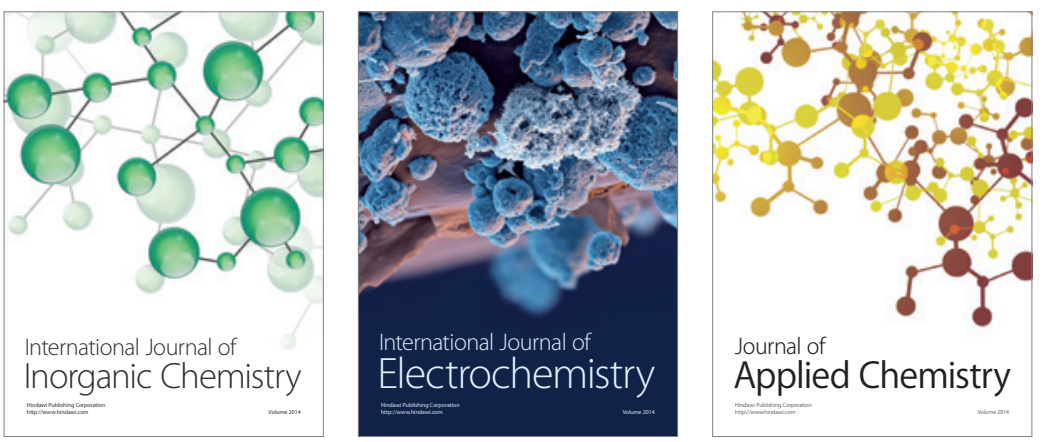

Journal of

Applied Chemistry
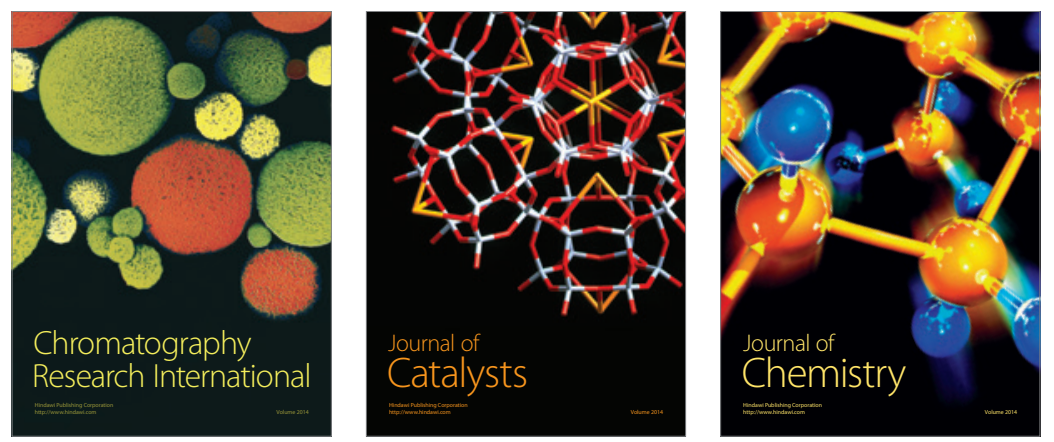
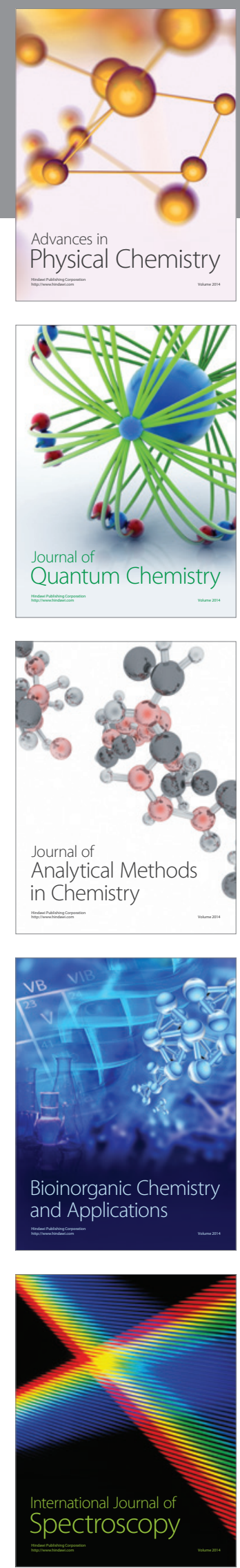